\title{
TRATAMIENTO QUIRÚRGICO DE LA ENFERMEDAD VALVULAR CARDIACA DE PREDOMINIO IZQUIERDO, EN UN HOSPITAL DE REFERENCIA NACIONAL DE LIMA, PERÚ
}

\author{
Julio C. Vasquez ${ }^{1, a}$, Ciro A. Barrantes ${ }^{2, b}$, Julio E. Peralta3,a, Luis E. Rojas 3 ,a
}

\section{RESUMEN}

Objetivos. Describir las características preoperatorias, intraoperatorias y posoperatorias de los pacientes con enfermedad valvular cardiaca de predominio izquierdo (EVCPI) tratados en un servicio de cirugía de tórax y cardiovascular de un hospital de referencia nacional; así como describir la ocurrencia de eventos tromboembólicos y hemorrágicos en estos pacientes. Materiales y métodos. Se realizó un estudio longitudinal retrospectivo en el que se incluyeron 185 pacientes operados entre 1999 y 2006 en el Hospital Nacional Dos de Mayo (Lima, Perú). Los pacientes fueron divididos en cuatro grupos: con comisurotomía mitral; con reemplazo valvular aórtico; con reemplazo valvular mitral y con doble reemplazo valvular. Se empleó la prueba $t$ de student, la prueba chi cuadrado, el análisis de varianza y la prueba de Bonferroni. El análisis de supervivencia tomó en cuenta los eventos tromboembolicos y hemorrágicos serios y se fijó un tiempo de seguimiento de seis años. Resultados. El tiempo de enfermedad promedio fue 4,6 años. La etiología más frecuente fue enfermedad reumática valvular $(74,6 \%)$. La mortalidad hospitalaria fue $3,8 \%$, siendo la causa de muerte más frecuente el síndrome de bajo gasto cardiaco con falla multiorgánica asociada. La incidencia de eventos isquémicos (trombosis o embolización) en pacientes que tuvieron reemplazo valvular a largo plazo (más de 6 meses) fue 3,2\%, y de eventos hemorrágicos fue 4,3\%. Conclusiones. Los resultados del tratamiento quirúrgico de EVCPI en el Perú son favorables. La tasa de complicaciones y mortalidad hospitalaria, así como los eventos tromboembólicos y hemorrágicos a largo plazo son comparables con los reportados en la literatura mundial.

Palabras clave: Enfermedades de las válvulas cardíacas; Prótesis valvulares cardíacas; Supervivencia; Tromboembolia; Anticoagulantes (fuente: DeCS BIREME).

\section{SURGICAL TREATMENT FOR LEFT-SIDED HEART VALVE DISEASE IN A NATIONAL REFERENCE HOSPITAL IN LIMA, PERU}

\begin{abstract}
Objectives. To describe the preoperative, intraoperative and postoperative characteristics of patients with left-sided heart valve disease treated in the thoracic and cardiovascular surgery service of a national reference hospital; as well as to describe the occurrence of thromboembolic and bleeding events in these patients. Materials and methods. A retrospective longitudinal study was carried out, which included 185 patients who underwert surgery between 1999 and 2006 at the Hospital Nacional Dos de Mayo (Lima, Peru). The patients were divided into 4 groups: patients with mitral commissurotomy; with aortic valve replacement; with mitral valve replacement and with double valve replacement. T-student test, Chi-square test, analysis of variance and Bonferroni test were used. The survival analysis took into account the severe thromboembolic and bleeding events and a follow-up period of 7 years was set. Results. The average durations of disease was 4.6 years. The most frequent etiology was rheumatic valve disease (74.6\%). Hospital mortality was $3.8 \%$, the most frequent cause of death was low cardiac output syndrome associated to multiple organ failure. The incidence of ischemic events (thrombosis or embolization) in patients who had a long-term valve replacement (more than 6 months) was 3.2\%, and the incidence of bleeding events was $4.3 \%$. Conclusions. The results of surgical treatment for left-sided heart valve disease in Peru are favorable. The rate of complications and hospital mortality rate, as well as the long-term thromboembolic and bleeding events are comparable to those reported in the world literature.
\end{abstract}

Key words: Heart valve diseases; Heart valve prosthesis; Survival; Thromboembolism; Anticoagulants (source: MeSH NLM).

Portneuf Medical Center. Idaho, EE. UU.

Hospital Nacional Guillermo Almenara. Lima, Perú.

Programa de Cirugía de Tórax y Cardiovascular "Efraín Montesinos Mosqueira", Hospital Nacional Dos de Mayo. Lima, Perú.

Médico, cirujano de tórax y cardiovascular; ${ }^{\mathrm{b}}$ médico, cardiólogo

Recibido: 15-04-13 Aprobado: 07-08-13

Citar como: Vasquez JC, Barrantes CA, Peralta JE, Rojas LE. Tratamiento quirúrgico de la enfermedad valvular cardiaca de predominio izquierdo, en un hospital de referencia nacional de Lima, Perú. Rev Peru Med Exp Salud Publica. 2013;30(3):415-22. 


\section{INTRODUCCIÓN}

En 1912 Theodore Tuffier realizó la primera operación para tratamiento de enfermedad valvular en un paciente de sexo masculino de 26 años con diagnóstico de estenosis aortica ${ }^{(1)}$. Desde entonces, se ha progresado mucho, sobre todo luego del advenimiento de la circulación extracorpórea gracias a la bomba de perfusión o “máquina corazón-pulmón”. En 1960, Harken realizó el primer implante valvular protésico exitoso ${ }^{(2)}$. Hoy en día esta es una operación realizada en todo el mundo.

La prevalencia de la enfermedad valvular reumática en países en desarrollo es significativa ${ }^{(3)}$; consecuentemente, en estos países existe una alta prevalencia de compromiso valvular. El compromiso patológico de las válvulas del lado izquierdo del corazón, es decir, de la válvula aortica y mitral, puede resultar en estenosis, insuficiencia o una forma mixta de ambas (conocida como doble lesión valvular). Cuando el daño es serio, generalmente se requiere reemplazo valvular ${ }^{(2)}$. En la actualidad existen dos tipos de válvulas protésicas: las mecánicas y la bioprótesis (biológicas). Las válvulas biológicas se emplean en pacientes con contraindicación de uso de warfarina, incluyendo mujeres en edad fértil debido a la posibilidad de teratogenicidad causada por este medicamento. Sin embargo, las válvulas biológicas, como la Carpentier-Edwards de pericardio porcino, tienen un deterioro acelerado en pacientes jóvenes con enfermedad valvular reumática. Es por ello que las válvulas mecánicas están recomendadas en pacientes jóvenes (menores de 65 años), dado que tienen más durabilidad que válvulas biológicas, y en pacientes que requieren anticoagulación oral crónica para manejo de fibrilación auricular.

En ocasiones es posible evitar el reemplazo valvular y, en lugar de ello, se realiza una reparación valvular. La comisurotomía mitral quirúrgica abierta es una opción utilizada en el tratamiento de estenosis mitral severa de origen reumático. Se utiliza cuando hay poca calcificación valvular, con poca distorsión o acortamiento de cuerdas tendíneas ${ }^{(4)}$. Su principal ventaja es que se evita implante valvular protésico y por ello, no requiere anticoagulación oral crónica.

En el Perú hay escasa información sobre el tratamiento quirúrgico de la enfermedad cardiaca valvular de predominio izquierdo (EVCPI). Al igual que en otros países en desarrollo, el número de estas operaciones es limitado, debido a su alto costo, su complejidad técnica y la necesidad de tener soporte tecnológico e infraestructura hospitalaria adecuados. El objetivo de la presente investigación es describir las características preoperatorias, intraoperatorias y posoperatorias de los pacientes con EVCPI tratados en un servicio de cirugía de tórax y cardiovascular de un hospital de referencia nacional, así como describir la ocurrencia de eventos tromboembólicos y hemorrágicos en estos pacientes.

\section{MATERIALES Y MÉTODOS}

Se realizó un estudio longitudinal retrospectivo en el Programa de Cirugía de Tórax y Cardiovascular "Efraín Montesinos Mosqueira" del Hospital Nacional Dos de Mayo (Lima, Perú). Para lo cual se incluyó a todos los pacientes con EVCPI en quienes se practicó alguna cirugía cardiaca abierta con circulación extracorpórea entre febrero 1999 a junio 2006. Se excluyó pacientes con patología compleja asociada. La población en estudio fue dividida en cuatro grupos, en función a la técnica quirúrgica empleada: comisurotomía mitral, reemplazo de válvula aórtica, reemplazo de válvula mitral y doble reemplazo valvular (aórtico y mitral). La indicación quirúrgica se tomó siguiendo patrones internacionales definidos, los cuales incluyen definición clínica, hemodinámica y ecocardiográfica de severidad de patología valvular (2). La recolección de la información se realizó a través de la revisión de historias clínicas.

\section{EVALUACIÓN PREQUIRÚRGICA}

Para el periodo evaluado el Programa de Cirugía de Tórax y Cardiovascular (PCTC) contempló que todos los pacientes tengan ecocardiografía transtorácica preoperatoria; en los casos en los que esta no fue concluyente, se realizó ecografía transesofágica. El cateterismo cardiaco se practicó en todo paciente mayor de 65 años y en aquellos entre 30 y 65 años en los que se debía conocer las presiones intracardiacas, en particular cuando se requirió cuantificar la presión pulmonar. Todos los pacientes recibieron en el periodo preoperatorio inmediato una línea venosa central y una línea arterial.

\section{TÉCNICA QUIRÚRGICA}

En el PCTC, las operaciones se realizaron bajo anestesia general y a través de esternotomía mediana. Se utilizó catéter de Swan-Ganz selectivamente en los pacientes con hipertensión pulmonar severa; se usó circulación extracorpórea con un flujo medio de 1,8 a 2,2 litros/minuto e hipotermia de 28 a $34{ }^{\circ} \mathrm{C}$. La canulación arterial se realizó en la aorta ascendente. Se usó canulación venosa en la orejuela de la aurícula derecha para reemplazo valvular aórtico aislado, y doble canulación (vena cava superior y vena cava inferior) para las operaciones que requirieron abordaje de la válvula mitral. Se administró cardioplegia hipotérmica con sangre, de manera anterógrada a través de las arterias coronarias, y esta se administró 
cada 20 a 30 minutos durante el período de crossclamp aórtico. En todos los pacientes se colocó un tubo de drenaje (vent) a través de la vena superior pulmonar derecha, dirigiéndolo hacia el ventrículo izquierdo. Asimismo, el abordaje de la válvula aórtica se realizó a través de aortotomía transversa, o a través de abordaje transpeptal (cuando el tamaño de la aurícula izquierda no permitió la adecuada exposición). Las válvulas protésicas aórtica y mitral fueron implantadas con suturas no absorbibles (Ethibond ${ }^{\circledast} 2 / 0$ ) reforzadas con pledgets, en tanto que la anuloplastía tricúspide se hizo con Prolene ${ }^{\circledR}$ 2/0 o 3/0 reforzada con pledgets.

\section{MANEJO POSOPERATORIO}

EI PCTC cuenta con una Unidad de Cuidados Intensivos a donde se traslada a los pacientes durante el posoperatorio para manejo multidisciplinario (cirujanos de tórax y cardiovascular, cardiólogos e intensivistas). Todos los pacientes recibieron tubos de drenaje mediastinal y pleural, los cuales fueron retirados cuando el drenaje fue menor a $200 \mathrm{~mL} /$ día y no hubiere fuga de aire. Los pacientes con fibrilación auricular crónica fueron mantenidos en anticoagulación crónica con warfarina indefinidamente. Los pacientes con implante de válvula bioprotésica (biológica) recibieron anticoagulación oral con warfarina solo por 6 meses luego de la operación. Los pacientes con válvulas mecánicas recibieron anticoagulación oral de por vida. El control de anticoagulación se hizo de manera ambulatoria, y se buscó mantener un INR de 2,0 a 3,0 para válvulas aorticas y 2,5 a 3,5 para válvulas mitrales. Todos los pacientes fueron vistos periódicamente en el consultorio del PCTC, con citas cada 1 a 6 meses.

\section{ANÁLISIS ESTADÍSTICO}

Se emplearon medidas de resumen para las variables en estudio, se calcularon frecuencias absolutas y relativas para las variables cualitativas, y la media y desviación estándar para las variables cuantitativas. Se utilizó la prueba $t$ de student para muestras independientes y pareadas, según fuese el caso. Para variables discretas se utilizó la prueba de chi cuadrado. Se utilizó el análisis de varianza entre dos o más grupos, y la prueba de Bonferroni para comparaciones múltiples. Se calculó la incidencia de eventos isquémicos (trombosis o embolización) y eventos hemorrágicos en los pacientes que recibieron reemplazo valvular. Se consideró como eventos tromboembólico a todo aquel que requirió hospitalización tales como accidente cerebrovascular isquémico embólico, trombosis de válvula implantada y tromboembolismo a sistema arterial periférico; y se consideró como eventos hemorrágico serio a todo aquel que requirió hospitalización, con documentación de INR elevado, tales como hemorragia digestiva, hematomas en partes blandas y hemorragia intraarticular. Se utilizó el paquete estadístico STATA Ver 12.0.

\section{CONSIDERACIONES ÉTICAS}

Se contó con la aprobación de la Oficina de Investigación y del Comité de Ética del Hospital Nacional Dos de Mayo.

\section{RESULTADOS}

Se encontraron 220 pacientes elegibles, se excluyeron cinco pacientes que tuvieron reemplazo de la raíz aórtica (operación de Bentall) para tratamiento de aneurisma de aorta ascendente; así como un paciente con reemplazo de válvula tricúspide; otro con plastía de válvula aórtica con cierre simultáneo de comunicación interventricular; tres pacientes con valvuloplastía mitral con anillo, quienes tenían válvula con hojas normales que no coaptaban por tener dilatación severa del anillo valvular, y 25 pacientes por no tener información clínica completa. Con lo cual 185 pacientes formaron parte del presente estudio. El $85,7 \%$ fueron mujeres, y el $60,5 \%$ procedian de Lima Metropolitana. Todos los pacientes fueron sintomáticos, y fue la disnea $(98,4 \%)$ el síntoma más frecuente. Tres pacientes, todos con estenosis mitral severa, tuvieron accidente cerebrovascular embólico preoperatorio asociado a fibrilación auricular. En el $74,6 \%$ de los casos la etiología de la patología valvular fue la enfermedad valvular reumática (Tabla 1).

En el $15,1 \%$ de ellos se practicó comisurotomía; en el $27 \%$, reemplazo valvular aórtico (RVA); en el $44,3 \%$, reemplazo valvular mitral (RVM), y en el $13,5 \%$, doble reemplazo valvular izquierdo (RVA+RVM). La indicación para $\mathrm{RVA}(\mathrm{n}=50)$ fue estenosis [22\% $(11 / 50)]$; insuficiencia [34\% (17/50)], y doble lesión [44\% (22/50)]. En el caso del RVM, la indicación fue estenosis [12,2\% (10/82)]; insuficiencia [8,5\% (7/82)], y doble lesión [79,3\% (65/82)]. Dos pacientes tuvieron como indicación quirúrgica la disfunción de válvula protésica mitral, que había desarrollado pannus y resultó en estenosis mitral severa. Para RVA+RVM $(n=25)$, la indicación fue estenosis mitral e insuficiencia aórtica [4\% (1/25)]; doble lesión mitral y estenosis aórtica [4\% (1/25)]; insuficiencia mitral y aórtica [20\% (5/25)]; doble lesión mitral e insuficiencia aórtica [36\% (9/25)], y doble lesión mitral y aórtica [36\% (9/25)].

Tres pacientes en quienes se practicó RVM, presentaron comisurotomía mitral de 10 a 12 años atrás, para tratamiento de estenosis mitral. Fue necesario realizar un puente aortocoronario (bypass) en cuatro pacientes para el tratamiento de enfermedad coronaria aterosclerótica simultáneamente con reemplazo valvular aórtico. En 43 de los pacientes que 
Tabla 1. Características preoperatorias de pacientes con intervención valvular cardiaca, Hospital Nacional Dos de Mayo, Lima, Perú. 1999-2006

\begin{tabular}{|c|c|c|c|c|c|}
\hline \multirow{3}{*}{ Características } & \multicolumn{4}{|c|}{ Tipo de intervención valvular cardiaca } & \multirow{3}{*}{$\mathbf{p}$} \\
\hline & \multirow{2}{*}{$\begin{array}{c}\begin{array}{c}\text { Comisurotomía } \\
(n=28)\end{array} \\
N .^{\circ}(\%)\end{array}$} & \multirow{2}{*}{$\begin{array}{c}\begin{array}{c}\text { RVA } \\
(n=50)\end{array} \\
N .^{\circ}(\%)\end{array}$} & \multirow{2}{*}{$\begin{array}{c}\text { RVM } \\
(\mathrm{n}=82) \\
\mathrm{N}^{\circ}(\%) \\
\end{array}$} & \multirow{2}{*}{$\begin{array}{c}\text { RVA + RVM } \\
(n=25) \\
N \cdot{ }^{\circ}(\%)\end{array}$} & \\
\hline & & & & & \\
\hline \multicolumn{6}{|l|}{ Sexo } \\
\hline Masculino & $4(14,3)$ & $39(77,6)$ & $21(25,7)$ & $16(64,0)$ & \\
\hline Femenino & $24(85,7)$ & $11(22,4)$ & $61(74,3)$ & $9(36,0)$ & $<0,01$ \\
\hline Edad (años)* & $37,7(13,0)$ & $44,7(19,0)$ & $38,0(14,3)$ & $34,3(11,3)$ & 0,02 \\
\hline $12-19$ & $2(7,1)$ & $5(10,0)$ & $11(13,4)$ & $2(8,0)$ & 0,02 \\
\hline $20-39$ & $15(53,6)$ & $17(34,0)$ & $37(45,1)$ & $15(60,0)$ & 0,02 \\
\hline $40-59$ & $10(35,7)$ & $15(30,0)$ & $27(32,9)$ & $8(32,0)$ & 0,02 \\
\hline 60 a más & $1(3,6)$ & $13(26,0)$ & $7(8,6)$ & $0(0)$ & 0,02 \\
\hline \multicolumn{6}{|l|}{ Características clínicas } \\
\hline Tiempo de enfermedad (años)* & $4,5(4,6)$ & $3,2(4,3)$ & $5,2(6,4)$ & $5,2(4,5)$ & 0,17 \\
\hline Disnea & $28(100)$ & $48(96,0)$ & $81(98,8)$ & $25(100)$ & 0,43 \\
\hline Dolor torácico & $12(42,9)$ & $25(50,0)$ & $33(40,2)$ & $12(48,0)$ & 0,71 \\
\hline Ortopnea & $3(10,7)$ & $14(28,0)$ & $26(31,7)$ & $9(36,1)$ & 0,13 \\
\hline Edema de miembros inferiores & $6(21,4)$ & $13(26,0)$ & $32(39,0)$ & $11(44,0)$ & 0,14 \\
\hline Hemoptisis & $10(35,7)$ & $3(6,0)$ & $13(15,9)$ & $6(24,0)$ & $<0,01$ \\
\hline Fumador & $1(3,6)$ & $4(8,0)$ & $4(4,9)$ & $1(4,0)$ & 0,8 \\
\hline Fibrilación auricular & $18(64,3)$ & $3(6,0)$ & $59(72,0)$ & $16(64,0)$ & $<0,01$ \\
\hline Fracción de eyección * & $62,2(6,4)$ & $57,0(10,9)$ & $59,1(8,7)$ & $58,0(9,8)$ & 0,14 \\
\hline \multicolumn{6}{|l|}{ Grado de insuficiencia cardiaca ** } \\
\hline I & $1(3,6)$ & $2(4,0)$ & $2(2,4)$ & $1(4,0)$ & 0,56 \\
\hline II & $15(53,6)$ & $20(40,0)$ & $42(51,2)$ & $10(40,0)$ & \\
\hline III & $12(42,9)$ & $24(48,0)$ & $36(43,9)$ & $14(56,0)$ & \\
\hline IV & $0(0)$ & $4(8,0)$ & $2(2,4)$ & $0(0)$ & \\
\hline \multicolumn{6}{|l|}{ Etiología } \\
\hline Reumática & $27(96,4)$ & $13(26,0)$ & $76(92,7)$ & $22(88,0)$ & $<0,01$ \\
\hline Degenerativa & $0(0)$ & $8(16,0)$ & $0(0)$ & $0(0)$ & \\
\hline Endocarditis & $1(3,57)$ & $11(22,0)$ & $1(1,2)$ & $3(12,0)$ & \\
\hline Congénita & $0(0)$ & $18(36,0)$ & $0(0)$ & $0(0)$ & \\
\hline Otro & $0(0)$ & $0(0)$ & $5(6,1)$ & $0(0)$ & \\
\hline
\end{tabular}

RVA: reemplazo valvular aórtico, RVM: reemplazo valvular mitral

* Media \pm desviación estándar

** Clasificación de New York Heart Association (NYHA)

recibieron el reemplazo valvular mitral, se realizó la anuloplastía modificada de DeVega para el tratamiento de insuficiencia tricúspide severa. Por otro lado, once pacientes requirieron trombectomía de aurícula izquierda, pues se halló un trombo organizado ocupando parte de ella.

En total se implantaron 75 válvulas aorticas, de las cuales el 92\% (69/75) fueron válvulas mecánicas y el $8 \%(6 / 75)$ fueron válvulas bioprotésicas. Los modelos de válvulas usadas en la posición aórtica fueron: St. Jude Medical bivalva [36\% (27/75)]; Medtronic Hall [26,6\% (20/75)] $(n=20)$; Carbomedics bivalva [18,6\% (14/75)], y otras [18,6\% (27/75)]. Por otro lado, se implantaron 107 válvulas mitrales, de las cuales el 90,7\% (97/107) fueron válvulas mecánicas y el 9,3\% (10/107) fueron válvulas bioprotésicas. Los modelos de las válvulas usadas en la posición mitral fueron: St. Jude Medical bivalva [37,4\% (40/107)]; Medtronic Hall [20,6\% (22/107)]; Carbomedics bivalva [18,7\% (20/107)], y otras [23,4\% (25/107)] $(n=25)$.
Los pacientes con doble reemplazo valvular tuvieron mayor duración de circulación extracorpórea (269 \pm 67 minutos) y clampado aórtico (214 \pm 35.3 minutos) que los pacientes que tuvieron otras operaciones $(p<0,01)$. No hubo diferencia en la cantidad de transfusión sanguínea según tipo de operación $(p=0,10)$, la cual tuvo un promedio de 1,8 \pm 1,8 unidades por paciente (Tabla 2). Se observó mayor uso de inotrópicos en el período posoperatorio inmediato en pacientes con doble reemplazo valvular (Tabla 3 ), lo cual fue significativo para dopamina, dobutamina y adrenalina $(p<0,05)$.

Se investigó la disminución de diámetro de aurícula izquierda en los pacientes que recibieron reemplazo valvular mitral y aquellos que recibieron comisurotomía. Las mediciones se obtuvieron con ecocardiografía transtorácica en el período preoperatorio y al menos 6 meses luego de la operación. El diámetro inicial de aurícula izquierda para el grupo de comisurotomía fue 
Tabla 2. Características intra y posoperatorias según tipo de operación valvular, Hospital Nacional Dos de Mayo, Lima, Perú. 1999-2006

\begin{tabular}{|c|c|c|c|c|c|}
\hline \multirow{3}{*}{ Características } & \multicolumn{4}{|c|}{ Tipo de intervención valvular cardiaca } & \multirow{3}{*}{$\mathbf{p}$} \\
\hline & $\begin{array}{c}\text { Comisurotomía } \\
(\mathrm{n}=28)\end{array}$ & $\begin{array}{c}\text { RVA } \\
(n=50)\end{array}$ & $\begin{array}{c}\text { RVM } \\
(n=82)\end{array}$ & $\begin{array}{c}\text { RVA + RVM } \\
(n=25)\end{array}$ & \\
\hline & Media \pm DE & Media \pm DE & Media \pm DE & Media \pm DE & \\
\hline Tiempo de circulación extracorpórea (minutos) & $102,9 \pm 43,9$ & $176,7 \pm 51,4$ & $162,2 \pm 63,3$ & $269 \pm 67,5$ & $<0,01$ \\
\hline Tiempo de clampado aórtico (minutos) & $72,7 \pm 32,0$ & $133,8 \pm 36,3$ & $115,6 \pm 39,0$ & $214,4 \pm 35,3$ & $<0,01$ \\
\hline Número de PG transfundidos intra y posoperatorios & $0,9 \pm 1,1$ & $2,0 \pm 1,9$ & $1,66 \pm 1,7$ & $2,1 \pm 2,1$ & 0,10 \\
\hline Duración de hospitalización (días) & $12,4 \pm 5,1$ & $13,9 \pm 6,2$ & $16,6 \pm 13,2$ & $16,0 \pm 8,7$ & 0,18 \\
\hline Tiempo en Unidad de Cuidados Intensivos (días) & $5,1 \pm 2,0$ & $6,2 \pm 3,0$ & $7,1 \pm 6,6$ & $7,7 \pm 6,1$ & 0,27 \\
\hline Tiempo en ventilación mecánica (horas) & $9,9 \pm 5,0$ & $13,2 \pm 13,3$ & $16,4 \pm 14,1$ & $21,4 \pm 27,3$ & $<0,01^{\star}$ \\
\hline
\end{tabular}

RVA: reemplazo valvular aórtico, RVM: reemplazo valvular mitral, PG: paquetes globulares

* Diferencia solo entre el grupo de comisurotomía y el grupo RVA + RVM

$5,4 \pm 1,1 \mathrm{~cm}$ (rango: 4,1-7,8) y el diámetro postoperatorio fue $5,1 \pm 0,9 \mathrm{~cm}$ (rango: $3,5-7,3$ ). Para el grupo que recibió reemplazo valvular mitral, el diámetro inicial de aurícula izquierda fue $6,5 \pm 1,6 \mathrm{~cm}$ (rango: 4,0-11,6) y el diámetro postoperatorio fue $5,8 \pm 1,5$ (rango: 2,5-10,4). Luego, se calculó la diferencia absoluta entre el diámetro inicial y final de aurícula izquierda, para los pacientes que tenían ambas mediciones $(n=64)$. La diferencia absoluta entre el diámetro pre y posoperatorio de la aurícula izquierda fue mayor $(p=0,02)$ en el grupo que recibió reemplazo valvular mitral $(0,8 \pm 1,2 \mathrm{~cm})$ comparado con comisurotomía mitral $(0,2 \pm 0,7 \mathrm{~cm})$.

Se comparó el promedio de clase NYHA para el periodo preoperatorio $(2,47 \pm 0,60)$ con el obtenido al menos 6 meses luego del alta $(1,19 \pm 0,5)$. Se encontró que hubo mejoría de un poco más de una clase funcional NYHA (ejemplo, de NYHA III a NYHA II) luego de la operación $(p<0,01)$.

\section{SEGUIMIENTO POSOPERATORIO Y MORTALIDAD}

No se encontró diferencias $(p=0,27)$ en el tiempo promedio de hospitalización que fue de 15,1 $\pm 10,2$ días, mientras que en cuidados intensivos fue de 6,7 $\pm 5,2$ días (Tabla 3 ). Sin embargo, sí hubo diferencia $(p<0,01)$ en el tiempo de ventilación mecánica, el cual fue mayor para pacientes con doble reemplazo valvular (21,4 $\pm 27,3$ horas).
Con relación a las complicaciones posoperatorias, siete pacientes $(13 \%)$ desarrollaron atelectasias, sin diferencias entre los grupos $(p=0,52)$, un paciente presentó neumonía $(0,5 \%)$. Nueve pacientes $(4,9 \%)$ requirieron reesternotomía para control de sangrado mediastinal. Dos $(1,1 \%)$ pacientes desarrollaron azotemia severa por más de 3 días, la cual se resolvió sin necesidad de diálisis. Dos $(1,1 \%)$ pacientes desarrollaron convulsiones sin aparente explicación, y presentaron tomografía cerebral negativa. Dos $(1,1 \%)$ pacientes tuvieron hemorragia digestiva alta que requirió transfusión sanguínea; la endoscopía reveló lesiones agudas de la mucosa gástrica. Un paciente $(0,5 \%)$ requirió intervención quirúrgica de emergencia para remover un balón de contrapulsación aórtico que quedó atrapado en la arteria iliaca, luego de que se rompiera espontáneamente. Un paciente $(0,5 \%)$ desarrolló insuficiencia respiratoria aguda y síndrome de distress respiratorio agudo del adulto, con recuperación favorable luego de recibir ventilación mecánica prolongada. Un paciente $(0,5 \%)$ tuvo infección esternal que requirió desbridamiento quirúrgico y curetaje, con buen resultado luego de terapia antibiótica prolongada. Ningún paciente tuvo accidente cerebrovascular posoperatorio.

La mortalidad intrahospitalaria fue del 3,8\% (7/185), no hubo diferencia en mortalidad según el tipo de operación

Tabla 3. Uso de inotrópicos en el periodo posoperatorio inmediato según tipo de operación valvular, Hospital Nacional Dos de Mayo, Lima, Perú. 1999-2006

\begin{tabular}{|c|c|c|c|c|c|c|c|c|c|c|}
\hline \multirow{3}{*}{$\begin{array}{l}\text { Uso de ino- } \\
\text { trópicos }\end{array}$} & \multicolumn{8}{|c|}{ Tipo de intervención valvular cardiaca } & \multirow{3}{*}{$\mathbf{p}_{1}$} & \multirow{3}{*}{$\mathbf{p}_{2}$} \\
\hline & \multicolumn{2}{|c|}{$\begin{array}{c}\text { Comisurotomía } \\
(n=28)\end{array}$} & \multicolumn{2}{|c|}{$\begin{array}{c}\text { RVA } \\
(\mathrm{n}=50) \\
\end{array}$} & \multicolumn{2}{|c|}{$\begin{array}{c}\text { RVM } \\
(n=82) \\
\end{array}$} & \multicolumn{2}{|c|}{$\begin{array}{c}\text { RVA + RVM } \\
(n=25) \\
\end{array}$} & & \\
\hline & N. ${ }^{\circ}(\%)$ & Días de uso* & N. ${ }^{\circ}(\%)$ & Días de uso* & N. ${ }^{\circ}(\%)$ & Días de uso* & N. ${ }^{\circ}(\%)$ & Días de uso* & & \\
\hline Dopamina & $10(35,7)$ & $0,9 \pm 1,9$ & $33(66,0)$ & $1,8 \pm 1,7$ & $52(63,4)$ & $2,1 \pm 2,4$ & $17(68,0)$ & $2,8 \pm 3,2$ & 0,03 & 0,03 \\
\hline Dobutamina & $18(64,2)$ & $1,7 \pm 2,0$ & $34(68,0)$ & $2,2 \pm 2,0$ & $59(71,9)$ & $2,3 \pm 2,1$ & $21(84,0)$ & $4,0 \pm 3,1$ & 0,40 & 0,05 \\
\hline Adrenalina & $3(10,7)$ & $0,2 \pm 0,8$ & $4(8,0)$ & $0,2 \pm 0,7$ & $5(6,1)$ & $0,1 \pm 0,7$ & $6(24,0)$ & $0,8 \pm 1,7$ & 0,06 & 0,02 \\
\hline Milrinona & - & - & $2(4,0)$ & $0,01 \pm 0,2$ & $6(7,3)$ & $0,1 \pm 0,4$ & $2(8,0)$ & $0,3 \pm 1,4$ & 0,43 & 0,21 \\
\hline
\end{tabular}

$\mathrm{p}_{1}$ : comparación entre el número de pacientes que usaron inotrópicos.

$\mathrm{p}_{2}$ : comparación entre el número de días de uso de inotrópicos.

RVA: reemplazo valvular aórtico, RVM: reemplazo valvular mitral

* Media \pm desviación estándar 
valvular $(p=0,87)$. De ellos, tres pacientes fallecieron dentro de las 72 horas luego de la operación, la cual fue considerada muerte temprana intrahospitalaria. En estos, el pH obtenido en análisis de gases arteriales menos de 4 horas después de la operación fue más acidótico que en los sobrevivientes $(7,25 \pm 0,03$ y $7,38 \pm 0,08$ respectivamente, $p<0,01)$. La causa de muerte intrahospitalaria más frecuente $[57,1 \%(4 / 7)]$ fue síndrome de bajo gasto cardiaco (shock cardiogénico), lo cual originó falla multiorgánica. En otros dos casos $[28,6 \%(2 / 7)]$ la causa de muerte fue sepsis.

No hubo diferencia en la mortalidad entre pacientes con diagnóstico preoperatorio de endocarditis y sin ella $(p=0,59)$. En pacientes con reemplazo valvular mitral aislado no se halló diferencia en la distribución de mortalidad entre los pacientes con o sin hipertensión pulmonar severa $(p=0,66)$. Tampoco se encontró diferencia respecto a la distribución de mortalidad por sexo $(p=0,22)$.

Entre los pacientes que recibieron reemplazo valvular, hubo 91 pacientes que fueron seguidos por un mínimo de 6 meses. De ellos, tres presentaron eventos isquémicos $(3,2 \%)$ y cuatro presentaron eventos hemorrágicos $(4,3 \%)$ durante el seguimiento. En el grupo de pacientes con reemplazo valvular, un paciente requirió reoperación luego de un año y medio para tratamiento de trombosis de válvula aortica protésica, con buen resultado y sin recurrencia. Un paciente requirió implante de marcapaso transvenoso permanente para tratamiento de bloqueo auriculoventricular de tercer grado. Dos pacientes desarrollaron tuberculosis pulmonar más de 2 años después de la operación valvular cardiaca y fueron curados con tratamiento específico.

\section{DISCUSIÓN}

La alta frecuencia de adultos jóvenes de nuestra muestra estaría relacionada con la alta frecuencia de enfermedad valvular reumática encontrada. Estos pacientes generalmente contraen fiebre reumática en la niñez o adolescencia y desarrollan síntomas entre los $20-40$ años de edad. En países en desarrollo, hasta 2 - $3 \%$ de niños en edad escolar tienen evidencia de enfermedad cardiaca reumática detectada por ecocardiografía, lo cual representa un problema de salud pública ${ }^{(3)}$. En nuestra serie, el tiempo promedio de enfermedad antes de la operación fue de casi 5 años. Aun cuando muchos de estos pacientes fueron diagnosticados previamente, pero por falta de recursos económicos no pudieron operarse oportunamente, lo cual habría condicionado la aparición de síntomas avanzados de algunas de las enfermedades valvulares estudiadas, como la hemoptisis, la que se relaciona generalmente con estenosis mitral ${ }^{(5)}$.
Por otro lado, la comisurotomía mitral quirúrgica abierta es una opción utilizada en el tratamiento de estenosis mitral de origen reumático ${ }^{(4)}$. Sin embargo, nosotros preferimos usar comisurotomía mitral solo para tratamiento aislado de estenosis mitral reumática. Ello debido a que si se utiliza en pacientes que requieren también reemplazo valvular aórtico, las complicaciones tromboembólicas y la sobrevida a largo plazo son semejantes a la obtenida con reemplazo valvular mitral, pero incrementa la necesidad de reoperación futura para reemplazo de válvula mitral ${ }^{(6)}$. Se ha descrito, además, que el tratamiento quirúrgico de la válvula mitral generalmente resulta en disminución del tamaño de la aurícula izquierda (7). En nuestros pacientes con reemplazo valvular mitral encontramos que tuvieron una mayor disminución absoluta de aurícula izquierda que los pacientes con comisurotomía.

La enfermedad valvular reumática es prácticamente inexistente en países desarrollados, pero aun relativamente frecuente en países en vías de desarrollo, como Perú. La enfermedad valvular reumática aórtica es tratada casi universalmente con reemplazo valvular. Sin embargo, en el caso de la válvula mitral, se puede intentar repararla sin necesidad de reemplazarla. Esto es controversial, pero la mayoría de series en la literatura muestran mejores resultados a largo plazo en pacientes con reemplazo de válvula mitral ${ }^{(8)}$.

La anticoagulación oral incrementa el riesgo de hemorragia severa, sobre todo cuando el INR es mayor de $5^{(9)}$. La frecuencia de eventos serios se ha reportado entre 1 y $2,5 \%$ por paciente-año, siendo el riesgo mayor durante los primeros meses luego de la operación ${ }^{(10,11)}$. En este estudio se encontró que luego de 6 meses, más del $95 \%$ de pacientes no ha presentado eventos serios, lo cual está dentro de lo reportado por la literatura. El tratamiento en estos casos consiste en interrupción inmediata de warfarina, y la administración de plasma fresco congelado y vitamina $\mathrm{K}$ por vía parenteral ${ }^{(12)}$, lo cual fue realizado en todos nuestros pacientes que presentaron esta condición. En pacientes con fibrilación auricular crónica, el uso de anticoagulación es mandatario para reducir el riesgo de tromboembolismo. Recientemente se han descrito resultados favorables con el procedimiento de Maze para eliminar la fibrilación auricular en pacientes con enfermedad valvular reumática de la válvula mitral. De igual modo, se ha descrito que el uso de crioablación es seguro y efectivo, en lugar de la técnica clásica de corte y sutura inicialmente descrita para esta operación ${ }^{(13)}$.

Con el uso de warfarina, el riesgo de tromboembolismo originado en válvulas mecánicas es de 1 a $2 \%$ por año, pero el riesgo es mayor sin warfarina ${ }^{(14)}$. Los pacientes 
con válvulas bioprotésicas (biológicas) generalmente no requieren anticoagulación oral, y el riesgo de tromboembolismo es de $0,7 \%$ por año en pacientes con ritmo sinusal ${ }^{(9)}$. Con ambos tipos de válvula (mecánica y biológica), el riesgo es mayor si se localiza en posición mitral. El riesgo de tromboembolismo es alto durante los primeros meses luego del implante valvular, pues la válvula aún no está cubierta de endotelio ${ }^{(15)}$. La presencia de fibrilación auricular también es un factor importante relacionado con tromboembolismo ${ }^{(16)}$. Nuestro análisis muestra que a los 6 meses, más del $95 \%$ de pacientes no han tenido eventos tromboembólicos, lo cual está dentro de lo esperado. En el caso de trombosis de válvula protésica, el tratamiento inicial debe ser terapia fibrinolítica con factor activador de plasminógeno (17). Sin embargo, cuando el trombo es muy grande o causa obstrucción, se debe proceder con tratamiento quirúrgico, como ocurrió con el único paciente que presento esta complicación.

La mortalidad operatoria inmediata es comparable con la reportada en la literatura: 3-8\% para reemplazo de válvula mitral; 7-12\% para reemplazo de válvula mitral, y 5-20\% para doble reemplazo valvular (aórtico y mitral) ${ }^{(18,19)}$. En el Perú hay escasa información publicada al respecto. En 2005, Bracamonte et al. presentaron 75 pacientes con reemplazo valvular aórtico operados en un periodo de 14 años (19912004). No se incluyó reoperaciones o cirugía "de rescate". La etiología fue reumática en $26 \%$ de los pacientes; por otro lado, $37 \%$ de los pacientes tuvieron puente aortocoronario simultáneo para tratamiento de enfermedad coronaria, y $15 \%$ tuvieron cirugía valvular en otra válvula cardiaca. La mortalidad hospitalaria fue de $5,3 \%{ }^{(20)}$.

En general, la tasa de mortalidad y mortalidad de pacientes con EVCPI es más alta que la de pacientes con enfermedad valvular degenerativa, a pesar de que en promedio son más jóvenes. Esto se debe probablemente a que se presentan tarde para atención médica con una enfermedad muy avanzada y dilatación masiva del corazón. Nuestros resultados son comparables a series similares publicadas en Brasil (21).

Se han descrito varios factores asociados a mortalidad en reemplazo valvular mitral, incluyendo sexo femenino ${ }^{(22)}$, en nuestra serie de 82 pacientes que tuvieron esta operación, todos los pacientes fallecidos fueron del sexo femenino. Aunque no se encontró diferencia significativa, esto puede haber sido consecuencia de falta de poder estadístico debido al tamaño limitado de nuestra población de estudio. Por otro lado, algunos estudios han mostrado asociación entre los valores de lactato sérico durante circulación extracorpórea ( $\geq 4 \mathrm{mmol} / \mathrm{L}$ ) o en el periodo posoperatorio inmediato ( $\geq 3 \mathrm{mmol} / \mathrm{L}$ ) y mortalidad en cirugía cardiaca ${ }^{(23,24)}$. En nuestra serie, el valor de lactato no estuvo disponible para la mayoría de pacientes porque no se midió rutinariamente. Por esta razón, buscamos sustitutos relacionados como $\mathrm{pH}$ y exceso (déficit) de base. Encontramos que valores bajos de $\mathrm{pH}$ dentro de las primeras 4 horas estuvo relacionado con mortalidad temprana ( $<72$ horas), al igual que déficit de base entre 4-16 horas. Esto puede estar relacionado a daño severo durante el periodo intraoperatorio o posoperatorio inmediato, con hipoperfusión de tejidos que resulta en acidosis sistémica.

Nosotros usamos anuloplastía modificada de DeVega para el tratamiento de insuficiencia tricúspide severa, pues tiene efecto duradero, sobre todo en pacientes jóvenes con diámetro de anillo tricuspideo mayor de $30 \mathrm{~mm}{ }^{(25)}$.

En el caso de doble reemplazo valvular (aórtico y mitral), se analizó un subgrupo $(n=8)$ que también recibió anuloplastía tricúspide modificada de DeVega para tratamiento de insuficiencia tricúspide severa. Estos pacientes tienen mayor riesgo de complicaciones perioperatorias debido a la complejidad técnica de la operación y pobre estado general. En este estudio se halló una mortalidad de $12,5 \%$, que está dentro de lo reportado en la literatura mundial $(8-31 \%){ }^{(26,27)}$.

Algunas limitaciones deben ser reconocidas, en primer lugar por ser un estudio retrospectivo y depende de la información obtenida en las historias clínicas, lo cual puede estar incompleto. No todos los pacientes tuvieron información completa de ecocardiografía pre y posoperatoria, lo cual limitó la potencia estadística del estudio. La información detallada sobre anticoagulación posalta es limitada y solo tenemos datos sobre INR y dosis de warfarina cada 1-6 meses, que se obtenían durante las visitas a nuestro consultorio externo. El tiempo de seguimiento de pacientes es relativamente corto debido a que las operaciones son recientes. Durante el seguimiento posalta, algunos pacientes fueron perdidos porque vivían fuera de Lima o porque decidieron no continuar seguimiento en nuestro hospital, sin embargo, se procuró contactarlos telefónicamente para obtener información sobre su estado actual.

En conclusión, el tratamiento quirúrgico de pacientes con EVCPI, operados en el Programa de Cirugía de Tórax y Cardiovascular del Hospital Nacional Dos de Mayo entre los años 1999 y 2006, tiene resultados favorables, con morbilidad y mortalidad comparables con las reportadas en la literatura mundial.

Agradecimientos: al Dr. Efraín Montesinos Mosqueira (19372007), fundador y primer cirujano jefe del Programa de Cirugía de Tórax y Cardiovascular del Hospital Nacional Dos de Mayo.

Contribuciones de autoría: JV, CB, JP participaron de la concepción del trabajo de investigación; JV y CB, del diseño y 
recolección de los datos; LR, de la recolección de los datos; JV, del análisis e interpretación de los datos y de la redacción del artículo; y JV, CB y JP, de la revisión crítica. Todos los autores aprobaron la versión final del artículo.
Fuente de financiamiento: autofinanciado.

Conflictos de interés: los autores niegan conflictos de interés.

\section{REFERENCIAS BIBLIOGRÁFICAS}

1. Stephenson LW. History of cardiac surgery. En: Cohn L, ed. Cardiac surgery in the adult. 3rd edition. New York: Mc Graw Hill; 2008. p. 3-28.

2. Bonow RO, Carabello BA, Kanu C, de Leon AC Jr, Faxon DP, Freed MD, et al. ACC/AHA 2006 guidelines for the management of patients with valvular heart disease: a report of the American College of Cardiology/American Heart Association Task Force on Practice Guidelines (writing committee to revise the 1998 Guidelines for the Management of Patients With Valvular Heart Disease): developed in collaboration with the Society of Cardiovascular Anesthesiologists: endorsed by the Society for Cardiovascular Angiography and Interventions and the Society of Thoracic Surgeons. Circulation. 2006;114(5):e84231.

3. Carapetis JR. Rheumatic heart disease in developing countries. N Engl J Med. 2007;357(5):439-41.

4. Choudhary SK, Dhareshwar J, Govil A, Airan B, Kumar AS. Open mitral commisurotomy in the current era: indications, technique and results. Ann Thorac Surg. 2003;75(1):41-6.

5. Buckhardt D, Hoffmann A, Kiowski W. Treatment of mitral stenosis. Eur Heart J. 1991;12 Suppl B:95-8.

6. Kuwaki K, Kawaharada N, Morishita K, Koyanagi T, Osawa H, Maeda T, et al. Mitral valve repair versus replacement in simultaneous mitral and aortic valve surgery for rheumatic disease. Ann Thorac Surg. 2007;83(2):558-63.

7. Cho DK, Ha JW, Chang BC, Lee SH, Yoon SJ, Shim CY, et al. Factors determining early left atrial reverse remodeling after mitral valve surgery. Am J Cardiol. 2008;101(3):374-7. doi: 10.1016/j.amjcard.2007.09.076.

8. Zhakkar M, Amirak E, Chan KMJ, Punjabi PP. Rheumatic mitral valve disease: current surgical status. Prog Cardiovasc Dis. 2009;51(6):478-81. doi: 10.1016/j. pcad.2008.08.008.

9. Bloomfield P, Wheatley DJ, Prescott RJ, Miller HC. Twelve-year comparison of a Bjork-Shiley mechanical heart valve with porcine bioprostheses. N Engl J Med. 1991;324(9):573-9.

10. Khan SS, Trento A, DeRobertis M, Kass RM, Sandhu M, Czer LS, et al. Twenty year comparison of tissue and mechanical valve replacement. J Thorac Cardiovasc Surg. 2001;122(2):257-69.

11. Ikonomidis JS, Kratz JM, Crumbley AJ, Stroud MR, Bradley SM, Sade RM, et al. Twenty year experience with the St. Jude Medical mechanical valve prosthesis. J Thorac Cardiovasc Surg. 2003;126(6):2022-31.

12. Ansell J, Hirsh J, Poller L, Bussey H, Jacobson A, Hylek E. The pharmacology and management of the vitamin K antagonists: the seventh ACCP Conference on Antithrombotic and Thrombolytic Therapy. Chest. 2004;126(3 Suppl):204S-233S.

13. Baek MJ, Na CY, Oh SS, Lee CH, Kim $\mathrm{JH}$, Seo HJ, et al. Surgical treatment of chronic atrial fibrillation combined with rheumatic valve disease: effects of the cryomaze procedure and predictors for late recurrence. Eur J Cardiothorac Surg. 2006;30(5):728-36.

14. Vongpatanasin W, Hillis LD, Lange RA. Prosthetic heart valves. N Engl J Med. 1996;335(6):407-16.

15. Hirsh J, Fuster V, Ansell J, Halperin JL. American Heart Association/American College of Cardiology Foundation guide to warfarin therapy. J Am Coll Cardiol. 2003;41(9):1633-52.

16. De Santo LS, Romano G, Della Corte A, Tizzano F, Petraio A, Amarelli C, et al. Mitral mechanical replacement in young rheumatic women: analysis of long term survival, valve related complications and pregnancy outcomes over a 3707 patient year follow up. J Thorac Cardiovasc Surg. 2005;130(1):13-9.

17. Shapira Y, Herz I, Vaturi M, Porter A, Adler Y, Birnbaum Y, et al. Thrombolysis is an effective and safe therapy in stuck bileaflet mitral valves in the absence of high risk thrombi. J Am Coll Cardiol. 2000;35(7):1874-80.

18. Thourani VH, Weintraub WS, Craver JM, Jones EL, Mahoney EM, Guyton $\mathrm{R}$. Ten year trends in heart valve replacement operations. Ann Thorac Surg. 2000;70(2):448-55.

19. Taylor NE, O'Brien S, Edwards FH, Peterson ED, Bridges CR. Relationship between race and mortality after valve replacement surgery. Circulation. 2005;111(10):1305-12.

20. Bracamonte L, Aste H, Talledo O, Rodriguez A. Reemplazo valvular aórtico: resultados, opciones y expectativas en 75 casos consecutivos. Diagnostico (Peru). 2005;4(2):70-8.

21. Ribeiro GS, Tartof SY, Oliveira DWS, Guedes ACS, Reis MG, Riley LW, et al. Surgery for valvular heart disease: a population based study in a brazilian urban center. PLoS One. 2012;7(5):e37855. doi: 10.1371/journal.pone.0037855.

22. Song HK, Grab JD, O'Brien SM, Welke KF, Edwards F, Ungerleider RM. Gender differences in mortality after mitral valve operation: evidence for higher mortality in perimenopausal women. Ann Thorac Surg. 2008;85(6):2040-4. doi: 10.1016/j.athoracsur.2008.02.082.

23. Maillet JM, Le Besnerais P, Cantoni M, Nataf P, Ruffenach A, Lessana A, et al. Frequency, risk factors and outcome of hyperlactatemia after cardiac surgery. Chest. 2003;123(5):1361-6.

24. Demers P, Elkouri S, Martineau R, Couturier A, Cartier R. Outcome with high blood lactate levels during cardiopulmonary bypass in adult cardiac operations. Ann Thorac Surg. 2000;70(6):2082-6.

25. Tager R, Skudicky D, Mueller U, Essop R, Hammond G, Sareli P. Long term follow up of rheumatic patients undergoing left sided valve replacement with tricuspid annuloplasty: validity of preoperative echocardiographic criteria in the decision to perform tricuspid annuloplasty. Am J Cardiol. 1998;81(8):1013-6.

26. Han QQ, Xu ZY, Zhang BR, Zou LJ, Hao $\mathrm{JH}$, Huang SD. Primary triple valve surgery for advanced rheumatic heart disease in mainland China: a single center experience with 871 clinical cases. Eur J Cardiothorac Surg. 2007;31(5):845-50.

27. Alsoufi B, Rao V, Borger MA, Maganti M, Armstrong S, Feindel CM, et al. Short and long term results of triple valve surgery in the modern era. Ann Thorac Surg. 2006;81(6):2172-7.

Correspondencia: Julio Vásquez. Dirección: 777 Hospital Way. Pocatello, ID 83201, USA

Teléfono: 208-239-2580

Correo electrónico: juliov@portmed.org 